\title{
Ion-Beam-Induced Luminescence Analysis of $\beta$-SiAlON:Eu Scintillator under Focused Microbeam Irradiation
}

\author{
Raj Kumar Parajuli, ${ }^{1}$ Wataru Kada,${ }^{1 *}$ Shunsuke Kawabata, ${ }^{1,2}$ Yoshinori Matsubara, ${ }^{1}$ \\ Makoto Sakai, ${ }^{3}$ Kenta Miura, ${ }^{1}$ Takahiro Satoh, ${ }^{2}$ Masashi Koka, ${ }^{2}$ \\ Naoto Yamada, ${ }^{2}$ Tomihiro Kamiya, ${ }^{2}$ and Osamu Hanaizumi ${ }^{1}$ \\ ${ }^{1}$ Graduate School of Science and Technology, Gunma University, 1-5-1 Tenjin cho Kiryu, Gunma 376-8515, Japan \\ ${ }^{2}$ Takasaki Advanced Radiation Research Institute (TARRI), Japan Atomic Energy Agency (JAEA), \\ 1233 Watanuki, Takasaki, Gunma 370-1292, Japan \\ ${ }^{3}$ Graduate School of Medicine, Gunma University, 3-39-22 Showa-machi, Maebashi, Gunma 371-8511, Japan
}

(Received January 4, 2016; accepted May 18, 2016)

Keywords: $\beta$-SiAlON, IBIL, radiation hardness, $\mathrm{ZnS}: \mathrm{Ag}$, focused microbeam

The scintillation properties and radiation durability of $\beta$-SiAlON:Eu were evaluated under focused microbeam irradiation conditions using $3 \mathrm{MeV}$ protons. In situ observation of scintillation from $\beta$-SiAlON:Eu was monitored using ion-beam-induced luminescence (IBIL) and compared with that from $\mathrm{ZnS}: \mathrm{Ag}$ scintillators. A comparison of the spectra of IBIL from both scintillators shows that the intensity of IBIL was analogous at different peak wavelengths of $545 \mathrm{~nm}$ for $\beta$-SiAlON:Eu and $450 \mathrm{~nm}$ for $\mathrm{ZnS}: \mathrm{Ag}$ under the same irradiation conditions. Better radiation hardness towards focused proton microbeam irradiation was observed for the $\beta$-SiAlON:Eu scintillator when continuous measurements by IBIL were used. A decay constant of approximately $1.11 \times 10^{16}$, which is two orders of magnitude higher than that for $\mathrm{ZnS}: \mathrm{Ag}$, was obtained for the $\beta$-SiAlON:Eu scintillator for focused proton microbeam irradiation. IBIL was also capable of visualizing a previously damaged area of a $\mathrm{ZnS}: \mathrm{Ag}$ scintillator, which corresponds to the focused beam scanning area of $100 \times 100 \mu \mathrm{m}^{2}$. Meanwhile, the irradiated region was not significantly distinguishable from the nonirradiated region on the $\beta$-SiAlON:Eu scintillator under the same beam fluence. These results suggest that $\beta$-SiAlON:Eu could be an ideal candidate scintillator for convenient ionized particle beam monitoring and a diagnostic tool for focused and intense beam fluence conditions up to $10^{16} \mathrm{ions} / \mathrm{cm}^{2}$.

\section{Introduction}

The radiation hardness of ionization particle detectors is a key issue for accelerator experiments, particularly for the utilization of focused ion microbeam probes for micometer-sized material modifications $^{(1,2)}$ or microanalysis..$^{(3,4)}$ Irradiation by a focused microbeam significantly degraded the response of particle detectors because of its intense energy transfer to the detector body. ${ }^{(5,6)}$ Thus, scintillators are one of the candidates for a detector that can be conveniently renewed after irradiation. ${ }^{(7,8)}$ However, it is ideal to avoid significant degradation in the light emission from scintillators during measurement to maintain the accuracy of the measurement. ${ }^{(9)}$ For such a purpose, various types of scintillators have been evaluated for ionized radiation monitoring. ${ }^{(10)}$ Among the various scintillators,

*Corresponding author: e-mail: kada.wataru@gunma-u.ac.jp 
silver-activated zinc sulfide ( $\mathrm{ZnS}: \mathrm{Ag}$ ) is quite popular for convenient $\alpha$-particle or ionized particle beam monitoring. ${ }^{(1-13)}$ The form of the powdered structure of $\mathrm{ZnS}: \mathrm{Ag}$ is also beneficial for ionized particle detection because of its high sensitivity to primary particles distinct from the background. ${ }^{(14)}$ Much effort is continuously made in the development of various scintillators with properties superior to those of $\mathrm{ZnS}: \mathrm{Ag}$. However, the high availability and low cost of $\mathrm{ZnS}: \mathrm{Ag}$ is an additional advantage, and it is still commonly used since it was first developed. However, sulfide-based phosphors have low chemical stability, causing their usefulness to depend on temperature and their degradation to occur because of radiation exposure. Therefore, it is necessary to find alternative candidates for scintillators for intense ion beam irradiation with performance comparable or superior to that of $\mathrm{ZnS}: \mathrm{Ag}$.

On the other hand, there is growing interest in high-luminescent-yield light conversion substrates for multiphosphor-converted white-light-emitting diodes (LEDs). ${ }^{(15)}$ Among candidate substrates, $\beta$-SiAlON:Eu ceramics are phosphors with excellent luminescence properties and outstanding thermal and chemical stability ${ }^{(16)}$ because of their hexagonal crystal structure equivalent to that of substituted $\beta$-phase silicon nitride $\left(\beta-\mathrm{Si}_{3} \mathrm{~N}_{4}\right)$ ceramics. ${ }^{(17)}$ Low thermal quenching would be delivered by doping with europium $(\mathrm{Eu})$, and the luminescence properties of the phosphor are maintained at even high temperatures of approximately $150{ }^{\circ} \mathrm{C} .{ }^{(18)}$ Because of its high efficiency and crystal structure, $\beta$-SiAlON:Eu is expected to be a successful candidate as an alternative scintillator to $\mathrm{ZnS}: \mathrm{Ag}$ for the convenient beam monitoring of intense ionized beam irradiation. Since the powders are originally fabricated for mass production, $\beta$-SiAlON:Eu is very promising because of its wide availability at a reasonable cost if its basic properties as a scintillator could be established by comparing its operation with conventionally available scintillators.

In this study, ion-beam-induced scintillation and the radiation hardness of $\beta$-SiAlON:Eu were preliminarily evaluated under focused proton microbeam irradiation conditions. To obtain the luminescence spectrum of a $\beta$-SiAlON:Eu scintillator, an ion-beam-induced luminescence (IBIL) analysis system using a focused proton microbeam was employed. The IBIL system was developed on the same microbeam line as a proton beam writing (PBW) system, which is capable of irradiating a target with any desired two dimensional patterns. The powders of $\beta$-SiAlON:Eu originally designed as the substrate of LEDs were processed to be fixed on the irradiation. Under focused proton beam irradiation conditions, the scintillation response was observed and compared with that of a commercially available $\mathrm{ZnS}: \mathrm{Ag}$ scintillator. IBIL was continuously monitored from both $\beta$-SiAlON:Eu and $\mathrm{ZnS}: \mathrm{Ag}$ scintillators to obtain the relative radiation tolerability with a beam fluence up to $10^{16}$ ions $/ \mathrm{cm}^{2}$. The results obtained in this study suggested that $\beta$-SiAlON:Eu was capable of being used as a beam-monitoring tool for focused microbeam irradiation conditions, and it was more durable in higher beam fluence than the conventional standard ZnS:Ag scintillator.

\section{Materials and Methods}

\subsection{Preparation of IBIL target of $\beta$-SiAION:Eu}

A focused proton microbeam irradiation experiment on $\beta$-SiAlON:Eu was performed on a microbeam line of the $3 \mathrm{MV}$ single-ended accelerator at Takasaki Advanced Radiation Research Institute (TARRI), Japan Atomic Energy Agency (JAEA). ${ }^{(19)}$ The sample was prepared on a sample holder used in in-air particle-induced X-ray emission (PIXE) analysis. ${ }^{(20)}$ A powdered sample of $\beta$-SiAlON:Eu (DENKA GR-200) with a mean particle diameter of $21 \mu \mathrm{m}^{(16)}$ was employed for the 
irradiation. $\beta$-SiAlON:Eu powders were mixed with water and then homogeneously spread on a polyimide film at a thickness of approximately $7 \mu \mathrm{m} .{ }^{(20)}$ This film was attached to the sample holder for PIXE analysis and also worked as a beam extraction window for the external proton beam irradiation. To exclude the effect of auto-fluorescence from the polyimide film, another sample holder of aluminum plate was also used for the irradiation. Under both conditions, the water was removed by drying at room temperature (RT) for $10 \mathrm{~min}$. This procedure allowed us to fix the scintillator without any adhesives between the sample holders and the scintillators.

\subsection{IBIL analysis}

Figure 1 shows the analysis system for IBIL, which consists of simple microoptics. ${ }^{(21)}$ A focused proton microbeam with energy up to $3 \mathrm{MeV}$ was employed for IBIL measurements on the SiAlON scintillator in conjunction with the micrometer-scale patterned irradiation technique of a PBW system. ${ }^{(22)}$ The scanning pattern of the proton microbeam was controlled by electrostatic $X-Y$ scanners. The PBW system was capable of irradiating the sample with desired two-dimensional patterns over a maximum scanning area of $800 \times 800 \mu \mathrm{m}^{2}$.(23) Since the IBIL optics shared its focal point with that of the proton microbeam, IBIL from the irradiated area was collected separately from the background from visible lights in the environment with a high signal-to-noise $(\mathrm{S} / \mathrm{N})$ ratio. ${ }^{(24)}$ IBIL spectroscopy was performed using an electrically cooled back-thinned charge-coupled device (CCD) spectrometer (Solid Lambda CCD, Spectra Co., Ltd.) with an effective wavelength from 200 to $980 \mathrm{~nm} .{ }^{(25)}$ Continuous IBIL measurement was achieved by repeatedly acquiring spectra at a high rate with a minimum measurement time of $19 \mathrm{~ms}$. Although the CCD spectrometer cannot obtain the absolute emission rate of IBIL photons, changes in the chemical composition of the target appear as changes in the structure of the IBIL spectrum. ${ }^{(26)}$ For comparison of IBIL intensity changes and IBIL imaging, the spatial resolution is assumed to correspond to the typical diameter of the focused microbeam (approximately $1 \mu \mathrm{m}$ ), ${ }^{(27)}$ which was evaluated from the secondary microscopic image of the copper grid for transmission electron microscope (TEM) application. ${ }^{(28)}$

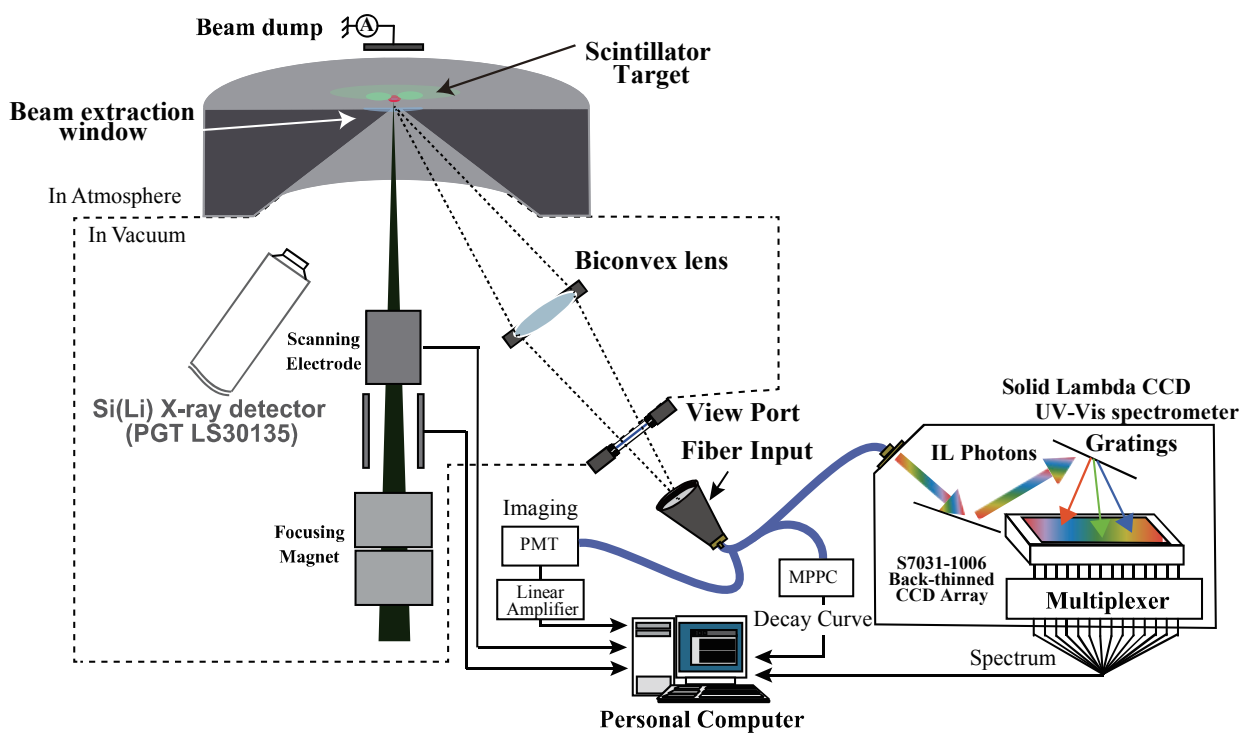

Fig. 1. (Color online) Schematic of experimental setup of IBIL analysis system for the evaluation of radiation hardness of a $\beta$-SiAlON:Eu scintillator. 
Since the spectrometer is not sensitive to the individual IBIL incident photons, it could be replaced by a photon-counting-mode photomultiplier (PMT, Hamamatsu Photonics; H10682-210) and a Geiger-mode avalanche photodiode (APD)-based multipixel photon counter (MPPC, Hamamatsu Photonics C10507-11-100U) for photon counting of the IBIL. Among the detectors used in this experiment, MPPC had the fastest response; therefore, the degradation of IBIL could be visualized with this detector, while PMT had the highest sensitivity to individual IBIL photons. Also, IBIL imaging was accomplished with the photon counting mode PMT to visualize the degraded region with the highest contrast within this experimental setup.

\section{Results and Discussion}

\subsection{Comparison of IBIL spectra of ZnS:Ag and B-SiAION:Eu}

The IBIL spectra of (a) ZnS:Ag and (b) $\beta$-SiAlON:Eu induced by focused $3 \mathrm{MeV}$ proton microbeam were obtained individually and reported as shown in Fig. 2. The IBIL was excited by protons with a variable beam current of approximately $1 \mathrm{pA}$. A CCD-based spectrometer was employed for the comparison of the spectra at the beginning of the irradiation. The intensity of the IBIL was similar for the two scintillators, with different peak wavelengths for $\beta$-SiAlON:Eu and $\mathrm{ZnS}: \mathrm{Ag}$ exhibiting a broad band structure. The peak at around $545 \mathrm{~nm}$ was obtained for $\beta$-SiAlON:Eu, which was longer than that of $\mathrm{ZnS}: \mathrm{Ag}(450 \mathrm{~nm})$ under the same irradiation conditions. The peak wavelength and structure of the IBIL spectrum of $\beta$-SiAlON:Eu were similar to those of the cathodoluminescence spectrum of $\beta-\mathrm{SiAlON}: \mathrm{Eu} .{ }^{(29)}$ The similar intensities of IBIL from both thin-layered scintillators suggest that the $\beta$-SiAlON:Eu scintillator has a comparable light emission yield to that of the $\mathrm{ZnS}: \mathrm{Ag}$, although the absolute scintillation efficiency could not be evaluated from the configuration of the microoptics. Both IBILs had a similar full width at half maximum (FWHM) of approximately $45 \mathrm{~nm}$. It is also remarkable that there are small differences in the peak wavelength, which could also be an advantage of $\beta$-SiAlON:Eu, because most photon detectors have maximum quantum efficiency at wavelengths at or above $500 \mathrm{~nm} .^{(30)}$

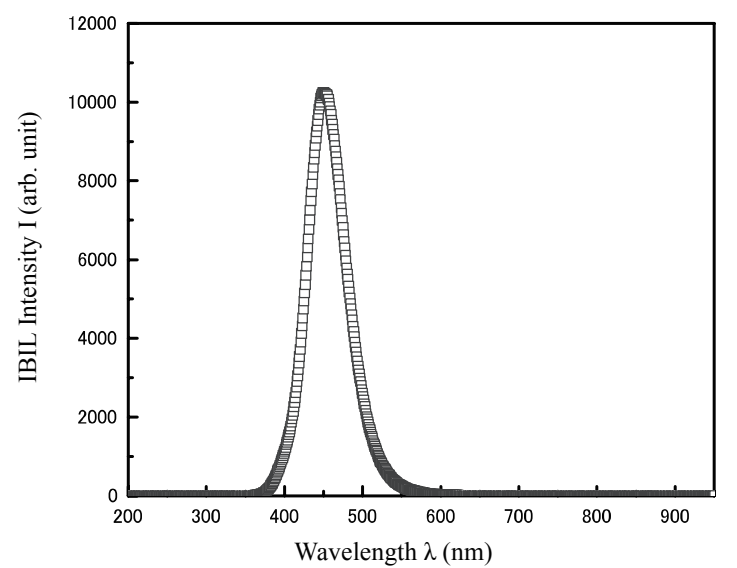

(a)

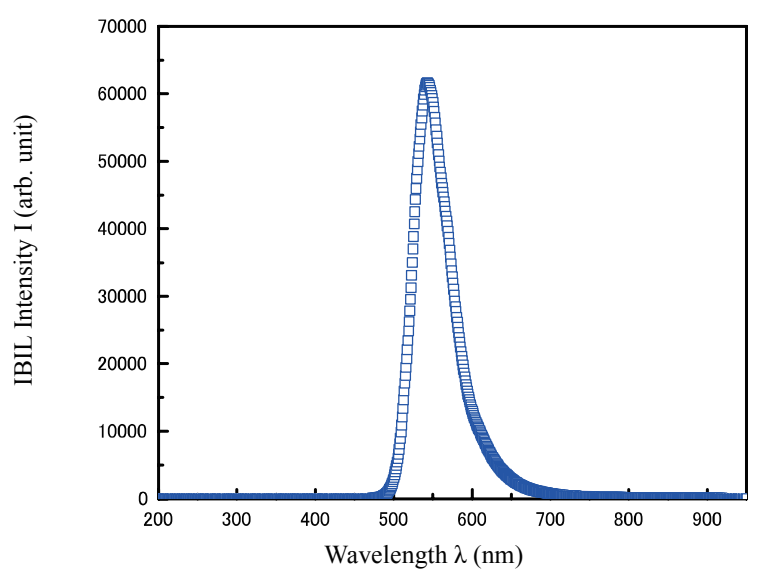

(b)

Fig. 2. (Color online) IBIL spectra obtained for (a) ZnS:Ag and (b) $\beta$-SiAlON:Eu excited by $3 \mathrm{MeV}$ proton beam irradiation. A peak at approximately $450 \mathrm{~nm}$ was obtained for IBIL from ZnS:Ag and one at approximately 545 $\mathrm{nm}$ for IBIL for $\beta$-SiAlON:Eu. 


\subsection{Continuous IBIL monitoring}

As irradiation continued, the structure of the IBIL spectrum of ZnS:Ag rapidly degraded, while the IBIL of $\beta$-SiAlON:Eu showed little fluctuation without significant degradation. Since the spectrometer is not sensitive to individual incident photons, the photodetectors of MPPC H10682210 were employed to compare IBIL intensity changes during focused microbeam exposure. The radiation resistivity of both scintillators is compared in Fig. 3 as the decay trend of IBIL for ZnS:Ag and $\beta$-SiAlON:Eu with respect to beam fluence and IBIL counts. A model using two decay factors $\left(F_{1}\right.$ and $\left.F_{2}\right)$ as expressed in Eq. (1) was employed to fit the IBIL intensity $I(F)$ (arb. unit) as a function of the beam fluence $F$ (ions $/ \mathrm{cm}^{2}$ ), where a constant factor $I_{0}$ was also included to express the baseline of the background and a slow decay component.

$$
I(F)=I_{0}+I_{1} \times \exp \left[-\left(F-F_{0}\right) / F_{1}\right]+I_{2} \times \exp \left[-\left(F-F_{0}\right) / F_{2}\right]
$$

A steep decay was observed for $\mathrm{ZnS}: \mathrm{Ag}$, whereas almost constant radiation tolerability was observed for the $\beta$-SiAlON:Eu scintillator. This fact was also cross-checked using the decay factors $\left(F_{1}\right.$ and $\left.F_{2}\right)$, which were determined for $\mathrm{ZnS}: \mathrm{Ag}\left(F_{1}=2.74 \times 10^{14}\right.$ and $\left.F_{2}=4.18 \times 10^{15}\right)$ and $\beta$-SiAlON:Eu $\left(F_{1}\right.$ and $\left.F_{2}=1.11 \times 10^{16}\right)$ from the fitting. The differences in the magnitudes of the decay factors correspond to the differences in the degradation of the IBIL responses of the two scintillators. The difference in the degradation of IBIL corresponds to the structural changes in the host crystal of the scintillator during ion bombardment. It appears that IBIL from ZnS:Ag and $\beta$-SiAlON:Eu showed fluctuation according to the fluctuation of the position and the current of the proton microbeam during the measurement. This experimental result suggests that the $\beta$-SiAlON:Eu scintillator has a better tolerance to radiation of the proton microbeam without any distortion caused by ion impact.

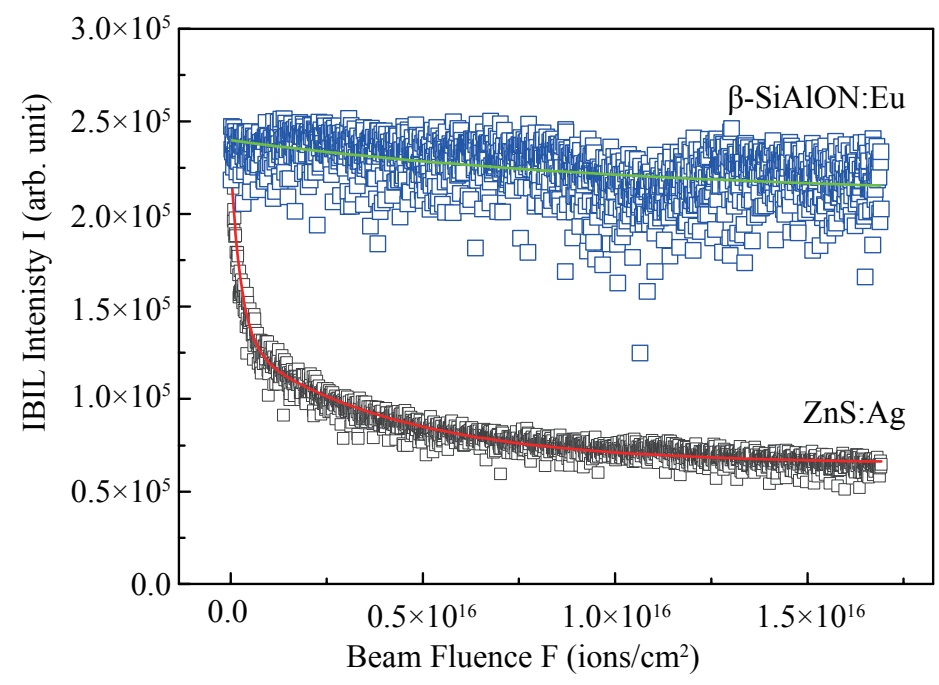

Fig. 3. (Color online) Comparison of decay scheme of IBIL for ZnS:Ag and $\beta$-SiAlON:Eu scintillator under intense fluence up to $1.5 \times 10^{16} \mathrm{ions} / \mathrm{cm}^{2}$. 


\subsection{In situ analysis by IBIL imaging}

After the intense irradiation of the $3 \mathrm{MeV}$ proton microbeam, in situ imaging of IBIL was performed using the same beam probe and photon-counting signals of H10682-210 PMT. Figure 4 shows comparisons of two-dimensional IBIL images for $\mathrm{ZnS}: \mathrm{Ag}$ and $\beta$-SiAlON:Eu scintillator targets before and after irradiation. In each image, the irradiated area of $100 \times 100 \mu \mathrm{m}^{2}$ size was indicated for a maximum scanning area of $200 \times 200 \mu \mathrm{m}^{2}$. Intense beam irradiation $(100 \times 100$ $\mu \mathrm{m}^{2}$ ) was applied with a beam current of $100 \mathrm{pA}$ for approximately $60 \mathrm{~min}$, and IBIL imaging was carried out with less than $1 \mathrm{pA}$. Only a few raster scans were carried out on the scintillators to avoid additional damage caused by IBIL imaging. IBIL was almost eliminated in the irradiated area on the $\mathrm{ZnS}: \mathrm{Ag}$ scintillator as seen in the comparison of images before and after irradiation. In contrast, the region cannot clearly be recognized in the comparison of two IBIL images for the $\beta$-SiAlON:Eu scintillator target. This result shows the usefulness of the $\beta$-SiAlON:Eu scintillator as a convenient particle detection material under beam irradiation conditions.

Since the $\beta$-SiAlON:Eu scintillator showed little degradation under continuous IBIL measurement, additional experiments on IBIL imaging for single particles of the $\beta$-SiAlON:Eu scintillator were carried out, as shown in Fig. 5, to evaluate the sustainability and uniformity of the IBIL from a single particulate target. The scanning beam of the proton microbeam has a scanning speed of several microseconds/pixel with a raster scan. Since there are no horizontal stripes on the IBIL image, IBIL induced by proton impact would have a decay time of less than several microseconds, which corresponded to the time spent scanning on the single point. During the

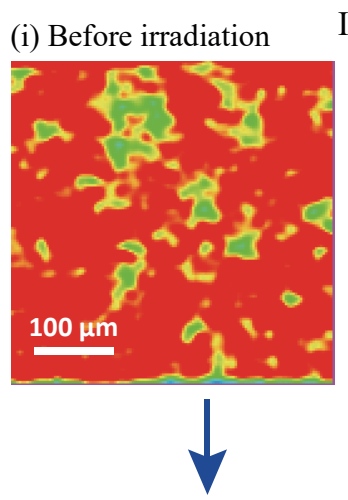

(ii) After irradiation

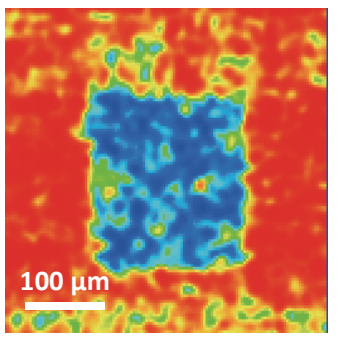

(a)

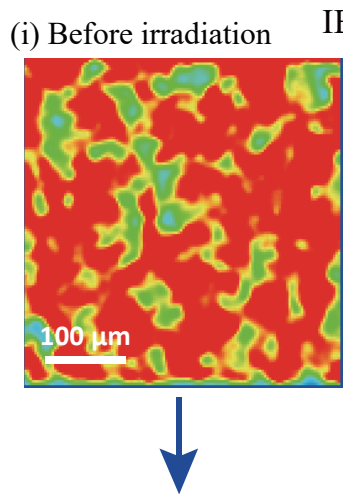

(ii) After irradiation

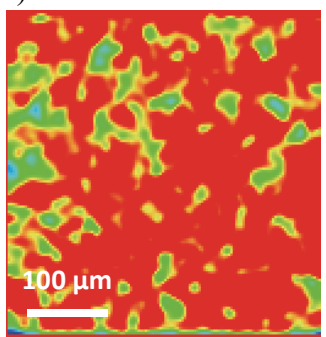

(b)
IBIL intensity

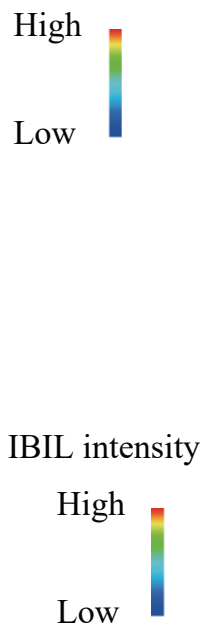

Low

Fig. 4. (Color online) IBIL imaging for (a) ZnS:Ag scintillator and (b) $\beta$-SiAlON:Eu scintillator. For both scintillators, IBIL images (i) before and (ii) after irradiation (intense irradiation area of $100 \times 100 \mu \mathrm{m}^{2}$ ) were visualized in IBIL images of the entire scanning area of $200 \times 200 \mu \mathrm{m}^{2}$. 


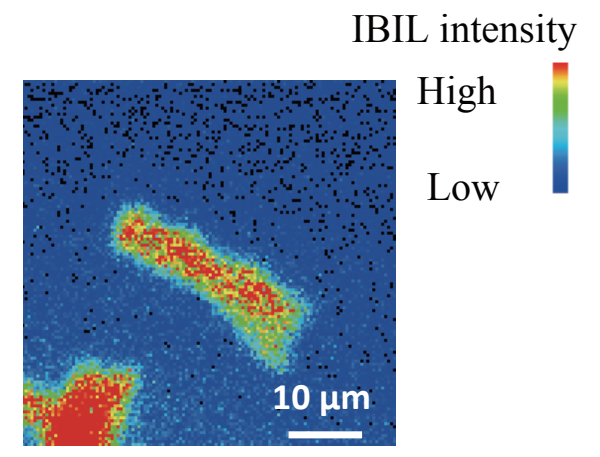

Fig. 5. (Color online) IBIL imaging for a single particle of the $\beta$-SiAlON:Eu scintillator.

irradiation, there are few fluctuations in IBIL intensity. Moreover, a uniform distribution of IBIL in the entire $\beta$-SiAlON:Eu particle was observed. These results suggest that the $\beta$-SiAlON:Eu scintillator may be applicable as a fluorescence staining material for a charged-particle microscope using electron, proton, or heavier particles, i.e., ion-photon emission microscopy (IPEM), ${ }^{(31)}$ where scintillators with high radiation hardness are required.

\section{Conclusions}

The scintillator properties and radiation resistance of $\beta$-SiAlON:Eu were preliminarily evaluated using a focused proton microbeam. IBIL spectra and quantitative measurements of IBIL intensity were collected for $\beta$-SiAlON:Eu and compared with $\mathrm{ZnS}: \mathrm{Ag}$ under intense proton irradiation. Comparison of IBILs revealed that similar light emission yields were obtained for $\mathrm{ZnS}: \mathrm{Ag}$ and $\beta$-SiAlON:Eu. Continuous observation of IBIL during intense proton irradiation revealed that the $\beta$-SiAlON:Eu scintillator has better radiation resistivity than the conventional standard of an $\mathrm{ZnS}$ :Ag scintillator. Postimaging of IBIL around the proton bombardment region suggested that the $\beta$-SiAlON:Eu scintillator is sustainable under harsh and intense ion beam irradiation conditions. Accurate measurement of the temperature dependence of IBIL for $\beta$-SiAlON:Eu is desirable to determine the threshold of the operational temperature range of this scintillator.

The remarkable properties of $\beta$-SiAlON:Eu suggest that it could be a successful candidate as a beam-monitoring tool for focused proton microbeams. Furthermore, single-particle imaging of $\beta$-SiAlON:Eu was also successfully demonstrated with IBIL imaging. Since little degradation was observed during irradiation, $\beta$-SiAlON:Eu scintillators are capable of being used as a fluorescence staining medium for an ion microscope if the mean diameter of the scintillator could be optimized. It is necessary to obtain the IBIL response of $\beta-\mathrm{SiAlON}: \mathrm{Eu}$ at high temperatures to determine its thermal response as a radiation detector.

\section{Acknowledgements}

This research was partially supported by a MEXT/JSPS Grant-in-Aid for Scientific Research 24710097 and 26706025. We sincerely thank Dr. Jun-ichi Susaki and Dr. Suzuya Yamada of Denka Co., Ltd. for providing $\beta$-SiAlON:Eu targets. This work was partially supported by the Cooperative Research Project of the Research Institute of Electronics, Shizuoka University. 


\section{References}

1 T. Kamiya, T. Satoh, M. Koka, and W. Kada: Nucl. Instrum. Methods Phys. Res., Sect. B 348 (2015) 4.

2 F. Watt, A. A. Bettiol, J. A. van Kan, E. J. Teo, and M. B. H. Breese: Int. J. Nanosci. 4 (2005) 269.

3 K. Ishii, A. Sugimoto, A. Tanaka, T. Satoh, S. Matsuyama, H. Yamazaki, C. Akama, T. Amartivan, H. Endoh, Y. Oishi, H. Yuki, S. Sugihara, M. Satoh, T. Kamiya, T. Sakai, K. Arakawa, M. Saidoh, and S. Oikawa: Nucl. Instrum. Methods Phys. Res., Sect. B 181 (2001) 448.

4 N. Fujita, K. Ishii, and H. Ogawa: Nucl. Instrum. Methods Phys. Res., Sect. B 269 (2011) 1023.

5 T. Satoh, M. Koka, W. Kada, A. Yokoyama, and T. Kamiya: Nucl. Instrum. Methods Phys. Res., Sect. B 332 (2014) 242.

6 G. Kalinka, M. Novák, A. Simon, Ž. Pastuović, M. Jakšić, and Á.Z. Kiss: Nucl. Instrum. Methods Phys. Res., Sect. B 267 (2009) 2203.

7 W. A. Hollerman, J. H. Fisher, D. Ila, G. M. Jenkins, and L. R. Holland: J. Mater. Res. 10 (1995) 1861.

8 A. Yokoyama, W. Kada, T. Satoh, M. Koka, S. Yamamoto, T. Kamiya, and W. Yokota: Nucl. Instrum. Methods Phys. Res., Sect. B 332 (2014) 334.

9 W. A. Hollerman, J. H. Fisher, G. A. Shelby, L. R. Holland, and G. M. Jenkins: IEEE Trans. Nucl. Sci. 38 (1991) 184.

10 M. Nishiura, N. Kubo, T. Hirouchi, T. Ido, T. Nagasaka, T. Mutoh, S. Matsuyama, M. Isobe, A. Okamoto, K. Shinto, S. Kitajima, M. Sasao, M. Nakatsuka, and K. Fujioka: Rev. Sci. Instrum. 77 (2006) 10E720.

11 T. Hirao, S. Onoda, M. Oikawa, T. Satoh, T. Kamiya, and T. Ohshima: Nucl. Instrum. Methods Phys. Res., Sect. B 267 (2009) 2216.

12 S. Matsuyama, K. Ishii, K. Watanabe, A. Terakawa, Y. Kikuchi, M. Fujiwara, H. Sugai, M. Karahashi, Y. Nozawa, S. Yamauchi, M. Fujisawa, M. Ishiya, T. Nagaya, R. Ortega, A. Carmona, and S. Roudeau: Nucl. Instrum. Methods Phys. Res., Sect. B 318 (2014) 32.

13 S. Calusi, E. Colombo, L. Giuntini, A. Lo Giudice, C. Manfredotti, M. Massi, G. Pratesi, and E. Vittone: Nucl. Instrum. Methods Phys. Res., Sect. B 266 (2008) 2306.

14 G. F. Knoll: Radiation Detection and Measurement (J Wiley, Tokyo, 2010) 4th ed., Chap. 8.

15 N. Hirosaki, R. J. Xie, K. Kimoto, T. Sekiguchi, Y. Yamamoto, T. Suehiro, and M. Mitomo: Appl. Phys. Lett. 86 (2005) 211905.

16 SiAlON Phosphors, ALONBRIGHT: Homepage of Denka Co., Ltd., http://www.denka.co.jp/eng/denzai/ product/filler/detail 002870.html (accessed December 2015).

17 K. H. Jack and W. I. Wilson: Nature 238 (1972) 28.

18 R. J. Xie, N. Hirosaki, H.-L. Li, Y. Q. Li, and M. Mitomo: J. Electrochem. Soc. 154 (2007) J314.

19 T. Kamiya, T. Suda, and R. Tanaka: Nucl. Instrum. Methods Phys. Res., Sect. B 104 (1995) 43.

20 T. Sakai, M. Oikawa, T. Sato, T. Nagamine, H. D. Moon, K. Nakazato, and K. Suzuki: Nucl. Instrum. Methods Phys. Res., Sect. B 231 (2005) 112.

21 W. Kada, T. Satoh, A. Yokoyama, M. Koka, and T. Kamiya: Nucl. Instrum. Methods Phys. Res., Sect. B 318 (2014) 42.

22 T. Kamiya, K. Takano, T. Satoh, Y. Ishii, H. Nishikawa, S. Seki, M. Sugimoto, S. Okumura, and M. Fukuda: Nucl. Instrum. Methods Phys. Res., Sect. B 269 (2011) 2184.

23 T. Sakai, R. Yasuda, H. Iikura, T. Nojima, M. Matsubayashi, W. Kada, M. Kohka, T. Satoh, T. Ohkubo, Y. Ishii, and K. Takano: Nucl. Instrum. Methods Phys. Res., Sect. B 306 (2013) 299.

24 W. Kada, A. Yokoyama, M. Koka, T. Satoh, and T. Kamiya: Int. J. PIXE 23 (2011) 47.

25 W. Kada, T. Satoh, A. Yokoyama, M. Koka, and T. Kamiya: Nucl. Instrum. Methods Phys. Res., Sect. B 332 (2014) 42.

26 W. Kada, A. Yokoyama, M. Koka, T. Satoh, and T. Kamiya: Int. J. PIXE 21 (2011) 1.

27 H. Watanabe and Y. Sudo: Nucl. Instrum. Methods Phys. Res., Sect. B 210 (2003) 1.

28 T. Sakai, Y. Naitoh, T. Kamiya, S. Matsuyama, K. Gotoh, S. Yotoka, H. Yamasaki, and K. Ishii: Biol. Trace Elem. Res. 71/72 (1999) 77.

29 N. Hirosaki, R. J. Xie, K. Kimoto, T. Sekiguchi, Y. Yamamoto, T. Suehiro, and M. Mitomo: Appl. Phys. Lett. 86 (2005) 211905.

30 Datasheet of Back-Thinned-Type CCD Area Image Sensor: Homepage of Hamamatsu Photonics K.K., http:// www.hamamatsu.com/resources/pdf/ssd/s7030-0906_etc_kmpd1023e.pdf (accessed December 2015).

31 P. Rossi, B. L. Doyle, J. C. Banks, A. Battistella, G. Gennaro, F. D. McDaniel, M. Mellon, E. Vittone, G. Vizkelethy, and N. D. Wing: Nucl. Instrum. Methods Phys. Res., Sect. B 210 (2003) 123. 